\title{
The utility of gadoteric acid in contrast-enhanced MRI: a review
}

\author{
This article was published in the following Dove Press journal: \\ Reports in Medical Imaging \\ 4 February 2014 \\ Number of times this article has been viewed
}

\section{Armando Tartaro \\ Marica Tina Maccarone \\ Department of Neuroscience, Imaging and Clinical Sciences, and Institute for Advanced Biomedical Technologies (ITAB), "G d'Annunzio" University, Chieti-Pescara, Italy}

Abstract: Gadoteric acid (Dotarem ${ }^{\circledR}$ ) is a macrocyclic, paramagnetic, gadolinium-based contrast agent. It is used in the magnetic resonance imaging (MRI) of the brain, spine, and associated tissues. Particularly, it is able to detect and visualize areas with disruption of the blood-brain barrier and/or abnormal vascularity. Gadoteric acid has been also approved for MR angiography of supraaortic vessels, cardiac MR (to detect myocardial infarctions), as well as whole-body MRI including abdominal, renal, pelvic, breast, and osteoarticular diseases. Cyclic chelates are more stable compared to linear chelates, and ionic chelates are more stable compared to nonionic chelates. Linear chelates have a greater likelihood of releasing free $\mathrm{Gd}^{3+}$ compared to cyclic chelates. Non-ionic chelates are more likely, compared to ionic chelates, to release $\mathrm{Gd}^{3+}$ from their chelates. Gadoteric acid is a cyclic ionic chelate and has the greatest kinetic stability among gadolinium-based contrast agents. In patients with chronic reduced kidney function, the use of gadolinium-based contrast agents leads to acute kidney injury and dialysis. The risk of acute kidney injury may increase with increasing dose of the contrast agents. Therefore, it is recommended to administer the lowest dose necessary for adequate imaging. The dose reduction allows protection the patients form potential risk of nephrogenic systemic fibrosis, a systemic reaction that is probably due to unbound $\mathrm{Gd}^{3+}$ ions deposited in body tissues. The dose of gadoteric acid should not exceed $0.1 \mathrm{mmol} / \mathrm{kg}$ body weight. More than one dose should not be used during a scan. Because of the lack of information on repeated administration, Dotarem ${ }^{\circledR}$ injections should not be repeated unless the interval between injections is at least 7 days. After administration, hemodialysis may not be useful to remove Dotarem ${ }^{\circledR}$ from the body. Dotarem ${ }^{\circledR}$ may allow the administration of a significantly lower dosage (a single dose of $0.1 \mathrm{mmol} / \mathrm{kg}$ ) compared to $0.5 \mathrm{mmol} / \mathrm{mL}$ non-protein-binding gadolinium compounds and achieving equivalent efficacy in the MRI diagnostic detection rate.

Keywords: contrast media, magnetic resonance imaging, gadoteric acid, macrocyclic gadolinium-based contrast agent

\section{Introduction}

Gadoteric acid (Dotarem ${ }^{\circledR}$, Guerbet, Roissy CdG Cedex, France) is a gadoliniumbased contrast agent (GBCA) for whole-body magnetic resonance imaging (MRI). Specifically, it is used to detect and visualize areas with disruption of the blood-brain barrier (BBB) and/or abnormal vascularity in MRI of the brain, spine, and associated tissues. Gadoteric acid is a macrocyclic paramagnetic molecule that develops a magnetic moment when placed in a magnetic field. The magnetic moment enhances the relaxation rates of water protons in its vicinity, leading to an increase in signal intensity (brightness) of tissues. The chemical name for gadoteric acid is D-glucitol, 1-deoxy-1(methylamino)-,[1,4,7,10-tetraazacyclododecane-1,4,7,10-tetraaceto(4-)-.kappa.N1,.
Correspondence: Armando Tartaro ITAB and Department of Neuroscience, Imaging and Clinical Sciences,

"G d'Annunzio" University,

Via dei Vestini, 33, Chieti 66013, Italy

Tel +3987 I 355690 I ext II

Fax +398713556930

Email tartaro@unich.it (c) (i) (9) 2015 Tartaro and Maccarone. This work is published by Dove Medical Press Limited, and licensed under Creative Commons Attribution - Non Commercial (unported, v3.0) BY License. The full terms of the License are available at http://creativecommons.org/licenses//by-nd/3.0/. Non-commercial uses of the work are permitted without any further permission from Dove Medical Press Limited, provided the work is properly attributed. Permissions beyond the scope of the License are administered by Dove Medical Press Limited. Information on
how to request permission may be found at: http://www.dovepress.com/permissions.php 


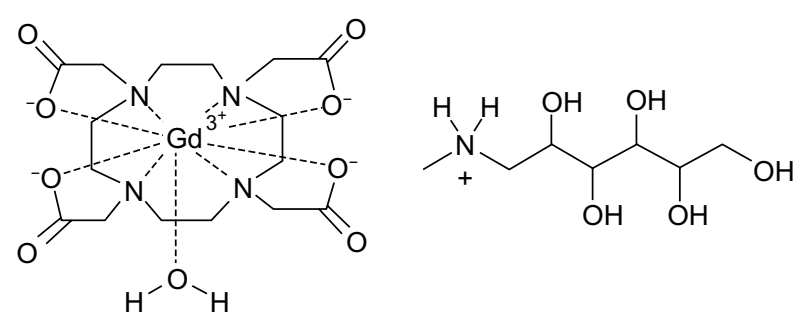

Figure I Structural formula of gadoteric acid in solution

kappa.N4,.kappa.N7,.kappa.N10,.kappa.O1,.kappa.O4,. kappa.O7,.kappa.O10]gadolinate(1-)(1:1). The formula weight is $753.9 \mathrm{~g} / \mathrm{mol}$ and its empirical formula is $\mathrm{C}_{23} \mathrm{H}_{42} \mathrm{O}_{13} \mathrm{~N}_{5} \mathrm{Gd}$ (anhydrous basis). The structural formula of gadoteric acid in solution is given below.

\section{Pharmacodynamics}

Gadoterate shortens the T1 (differences of the spin-lattice or longitudinal relaxation times) and T2 (differences in the spinspin or transverse relaxation time) relaxation times in target tissues. At recommended doses, the effect is observed with greatest sensitivity in the T1-weighted sequences. Gadoteric acid affects proton relaxation times and, consequently, the MR signal. The contrast obtained is characterized by the relaxivity of the gadoteric acid molecule. The relaxivity values for Dotarem ${ }^{\circledR}$ are similar across the spectrum of magnetic field strengths used in clinical MRI $(0.2-1.5 \mathrm{~T})$. The typical dose is $0.2 \mathrm{~mL} / \mathrm{kg}(0.1 \mathrm{mmol} / \mathrm{kg}$ iv $)$ with a rate of $2 \mathrm{~mL} / \mathrm{s}$ in adult patients. In patients $<2$ years old, the safety and efficacy of this contrast agent has been well established. The safety protocol must carefully consider the indications, and the dose should not exceed the recommended one for pediatric MRI.

In patients $>2$ years old, such as for adult patients, the dosage is $0.2 \mathrm{~mL} / \mathrm{kg}(0.1 \mathrm{mmol} / \mathrm{kg}$, iv $)$ with a rate of 1-2 mL/s. Therefore, for pediatric patients, including patients $<2$ years old, the recommended dose in the protocol is consistent with the currently proposed pediatric dose: $0.1 \mathrm{mmol} / \mathrm{kg}$ (ie, $0.2 \mathrm{~mL} / \mathrm{kg}$ ). Contrast-enhanced MR imaging (CE-MRI) can start immediately following the injection.

\section{Pharmacokinetics}

The pharmacokinetics of total gadolinium following an iv administration of a $0.1 \mathrm{mmol} / \mathrm{kg}$ dose of Dotarem ${ }^{\circledR}$ in normal subjects conforms to a two-compartment, open model with a mean distribution half-life (reported as mean \pm SD) of $7.1 \pm 5.2$ minutes and a mean elimination half-life of $91 \pm 14$ minutes in healthy volunteers. ${ }^{1}$

\section{Safety and contraindications}

The only contraindication is hypersensitivity to the GBCA. In addition, GBCAs increase the risk for nephrogenic systemic fibrosis (NSF) among patients with impaired renal function. Specifically, the risk of NSF is highest in case of chronic, severe kidney disease (ie, glomerular filtration rate [GFR] $<30 \mathrm{~mL} /$ $\min / 1.73 \mathrm{~m}^{2}$ ) or acute kidney injury. NSF may result in fatal or debilitating fibrosis, which affects the skin, muscle, and internal organs. $^{2}$

NSF affects mainly patients with end-stage renal disease, including patients on dialysis. It is characterized by the development of discolored skin plaques that can be itchy and painful. The extremities and the trunk are affected, but the head and neck are spared (with the exception of bilateral yellowed scleral plaques). Contractures of joints and complete loss of range of motion may occur. ${ }^{2}$

The disease severity varies among patients, and the fibrotic changes can be widespread, possibly affecting other organs including the liver, lungs, muscles, and heart. In some cases, NSF causes serious physical disability, which can lead to patients requiring a wheelchair. Mortality in patients with multisystem involvement has also been reported. ${ }^{2}$

Gadolinium is not water-soluble, and free gadolinium is highly toxic. The stability of GADAs seems to be an important factor in the pathogenesis of NSF. GBCA of low stability is likely to undergo transmetalation and release free $\mathrm{Gd}^{3+}$ ions. $\mathrm{Gd}^{3+}$ ions may deposit in tissues and attract circulating fibrocytes to initiate the process of fibrosis. Transmetalation refers to the replacement of the metal ion in the chelate by another ion. In vivo, endogenous ions (particularly zinc, which is readily available) can replace $\mathrm{Gd}^{3+}$ to form chelates, which are excreted in urine. The free $\mathrm{Gd}^{3+}$ cations become attached to endogenous anions, such as phosphate, hydroxide, carbonate, or citrate, to form insoluble salts that deposit in tissues. ${ }^{2}$

The epidemiology of NSF associated with GBCA suggests that the stability of the Gd chelates is likely to be an important factor in the pathogenesis of this condition. Extracellular GBCA includes all chelates containing $\mathrm{Gd}^{3+}$. Stability refers to the relative tendency of the $\mathrm{Gd}^{3+}$ to remain coordinated (attached) to the chelating agent. Free $\mathrm{Gd}^{3+}$ is highly toxic and can cause splenic degeneration, central lobular necrosis of the liver, enzyme inhibition, calciumchannel blocking, and a variety of hematological abnormalities beside NSF. Therefore, to avoid its toxic effects, it is crucially important that $\mathrm{Gd}^{3+}$ is strongly attached to a chelate. ${ }^{2}$ To overcome these problems, water-soluble chelates were designed with high $\mathrm{Gd}^{3+}$ affinity. There are nine coordination sites in most $\mathrm{Gd}^{3+}$ chelates. $\mathrm{Gd}^{3+}$ works as a 
contrast agent by disturbing the relaxation of surrounding water protons. In order for $\mathrm{Gd}^{3+}$ to interact with water, one of the coordination sites must be left free. This requirement decreases the binding affinity and allows some dissociation of the ion from its ligand. If the chelate is allowed to sit indefinitely in solution, then the binding affinity can be expressed as the thermodynamic stability. This concept is mathematically represented by the formula $K_{\text {therm }}=\left[\mathrm{Gd}^{3+/}-^{++}\right.$ chelate $] /\left[\mathrm{Gd}^{3+}\right] \times[$ chelate $]$, where a large value is associated with more stable binding and thus with less release of free $\mathrm{Gd}^{3+}$. A more physiologic stability constant is the conditional stability constant measured at $\mathrm{pH} 7.4$. The structure of the chelates determines these two stability constants. Cyclic chelates are more stable than linear chelates. The charge of the GBCA agent is also a factor in the stability, but has a lower impact than the structure of the ligand. Ionic chelates are more stable than nonionic chelates. ${ }^{3}$ In summary, linear chelates have a greater likelihood of releasing free $\mathrm{Gd}^{3+}$ than cyclic chelates, and nonionic chelates are more likely than ionic chelates to release $\mathrm{Gd}^{3+}$ from their chelates. The nonionic linear chelates gadodiamide and gadoversetamide as well as the ionic linear chelate gadopentetate dimeglumine are classified in the category of high-risk agents for NSF. The linear ionic chelate gadobenate dimeglumine and the linear nonionic chelates gadoxetic acid disodium and gadofosveset trisodium belong to the intermediate-risk category. Gadoteridol and gadobutrol are nonionic macrocyclic GBCAs whereas gadoteric acid is an ionic macrocyclic chelate. These three GBCAs belong to the low-risk category, but Dotarem ${ }^{\circledR}$ has the greatest kinetic stability of all the GBCA family. ${ }^{3}$

In vivo and in vitro clinical studies have demonstrated that gadodiamide $\left(\mathrm{Omniscan}^{\circledR}\right.$, GE Healthcare AS, Oslo, Norway) caused the highest increase in zinc in the urine, followed by gadopentetate dimeglumine (Magnevist ${ }^{\circledR}$, Bayer Schering Pharma AG, Berlin, Germany). The macrocyclic agent Dotarem ${ }^{\circledR}$ caused minimal zinc excretion. ${ }^{2}$ We recommend it for screening tests for acute kidney injury and other conditions that may reduce renal function for patients at risk for chronically reduced renal function (eg, age $>60$ years, hypertension, diabetes), GFR is estimated through laboratory testing. For patients at highest risk for NSF, the recommended dose should not be exceeded, and a sufficient period of time should be allowed for elimination of the drug before readministration.

In patients with chronically reduced renal function, acute kidney injury requiring dialysis has occurred with the use of GBCAs. ${ }^{4}$ The risk of acute kidney injury may increase with increasing dose of the contrast agent; thus the lowest dose necessary for adequate imaging should be administered. The dose reduction allows the reduction of the potential risk of NSF, a systemic reaction thought likely to be due to unbound $\mathrm{Gd}^{3+}$ ions deposited in body tissues (following contrast administration and in vivo dechelation). In the presence of unbound gadolinium, fibroblast activity appears to be activated, eventually leading to systemic sclerosis and, potentially, to death. It is widely accepted that the risk of NSF is greatly increased in patients with impaired renal function $\left(\right.$ GFR $\left.<30 \mathrm{~mL} / \mathrm{min} / 1.73 \mathrm{~m}^{2}\right){ }^{5}$ In these patients, repeated injection of a gadolinium chelate or a high-dose injection (or less commonly, even a single contrast injection) may increase the amount of retained free $\mathrm{Gd}^{3+}$ ion in the body, increasing the risk of $\mathrm{NSF}^{5}$

Transmetalation and spontaneous dissociation of GBCA, leading to the release of free $\mathrm{Gd}^{3+}$ cations, are likely to occur when the gadolinium chelate remains in the body for a long period, as is the case in patients with end-stage renal disease, including those on dialysis. It is reasonable to hypothesize that free $\mathrm{Gd}^{3+}$ cations become attached to endogenous anions, particularly phosphate, forming insoluble salts that deposit in tissues. These insoluble molecules are engulfed by local macrophages, which in turn release a range of cytokines, including the transforming growth factor-beta 1 (TGF- $\beta 1$ ), that attract circulating fibrocytes and initiate the process of fibrosis. ${ }^{2}$ There is evidence that tissue fibrosis in NSF is caused by circulating fibrocytes recruited from the circulation, rather than by proliferation of resident dendritic cells. ${ }^{2}$ In addition, hybridization studies have shown a marked increase in TGF- $\beta 1$ mRNA levels in the skin and fascia of patients with NSF. ${ }^{2}$ Risk minimization of NSF requires the screening of all patients for renal impairment by obtaining a history and/or laboratory tests, and following up renal function assessments for patients with a history of renal dysfunction. As the renal clearance of GBCAs may be impaired in the elderly, it is particularly important to screen patients aged 65 years and older for renal dysfunction. Dotarem $^{\circledR}$ should be used only in patients with severe renal impairment (GFR $<30 \mathrm{~mL} / \mathrm{min} / 1.73 \mathrm{~m}^{2}$ ) and in patients in the perioperative liver transplantation period (patients undergoing liver transplantation are at particular risk since the incidence of acute renal failure is high in this group), after careful risk/ benefit assessment and if the diagnostic information is essential and not obtainable with non-contrast-enhanced MRI. If it is necessary to use Dotarem ${ }^{\circledR}$, the dose should not exceed $0.1 \mathrm{mmol} / \mathrm{kg}$ body weight.

Hemodialysis shortly after Dotarem ${ }^{\circledR}$ administration may be useful in removing the GBCA from the body. There is no evidence to support the initiation of hemodialysis for 
the prevention or treatment of NSF in patients not already undergoing hemodialysis.

Patients on hemodialysis can be scheduled to have the dialysis session shortly after the MRI examination. Patients on peritoneal dialysis are at extra risk due to the slow elimination of GBCA. Multiple peritoneal dialysis should be encouraged to enhance the removal of the contrast molecules from the body. ${ }^{2}$

\section{Adverse reactions}

Anaphylactic and anaphylactoid reactions have been reported, which may involve cutaneous manifestations and/or cardiovascular and respiratory disease, including reports of patients experiencing cardiovascular collapse and death. Acute kidney injury requiring dialysis has occurred in patients with chronically reduced renal function. These effects have been attributed to GBCAs in general and not specifically to Dotarem ${ }^{\circledR} \cdot{ }^{6}$ Only $0.34 \%$ of total patients who underwent administration of GBCAs reported an adverse event. The most common symptoms were urticaria (wheals) $(<0.1 \%)$, pruritus $(<0.1 \%)$, increased sweating $(<0.1 \%)$, dizziness $(<0.1 \%)$, paresthesia $(<0.1 \%)$, nausea $(0.2 \%)$, vomiting $(0.1 \%)$, retching $(<0.1 \%)$, taste alteration $(<0.1 \%)$, feeling of warmth $(<0.1 \%)$, and increased sweating $(<0.1 \%) .{ }^{6}$ Other possible adverse effects of GBCAs are headache $(1 \%-10 \%, 1.5 \%$ pediatric), injection site pain $(0.4 \%)$, injection site coldness $(0.2 \%)$, burning sensation $(0.2 \%)$, feeling cold $(<0.2 \%)$, rash $(<0.2 \%)$, somnolence $(<0.2 \%)$, fatigue $(<0.2 \%)$, pain in extremities $(<0.2 \%)$, anxiety $(<0.2 \%)$, hypertension $(<0.2 \%)$, palpitations $(<0.2 \%)$, oropharyngeal discomfort $(<0.2 \%)$, increased serum creatinine $(<0.2 \%)$, and injection site reactions including site inflammation, extravasation, pruritus, and warmth $(<0.2 \%)$.

Even though the only contraindication to gadoteric acid administration is hypersensitivity to GDCAs, before administration all patients should be assessed for any history of prior reactions to contrast media or other allergens that required the administration of steroids or intubation, bronchial asthma, and/or allergic disorders, because this also helps in assessing the risk of hypersensitivity - these patients have an increased risk of hypersensitivity to GBCAs during and following administration, requiring observation for signs/symptoms of hypersensitivity. Pretreatment can be given to patients with allergies before the MRI examination, and it consists of an administration of sedatives, followed by $\mathrm{H} 1$-antihistamines, H2- antihistamines, and cortisone. ${ }^{6}$
Dotarem $^{\circledR}$ must be administered only in situations where trained personnel and therapies are promptly available for the treatment of hypersensitivity reactions, including personnel trained in resuscitation. During and following Dotarem ${ }^{\circledR}$ administration, patients should be observed for signs and symptoms of hypersensitivity reactions.

\section{Use in specific populations}

The use of GBCAs during pregnancy is not contraindicated, but the pregnancy category is $\mathrm{C}$ : "use with caution if benefits outweigh risks", as recommended by the European Society of Radiology guidelines, and based on the available evidence that GBCAs appear to be safe in pregnancy. ${ }^{7}$ Animal studies and human studies in pregnancy are not available. Limited published data on human exposure to other GBCAs during pregnancy did not show adverse effects in exposed neonates. Inadvertent human exposure during the first trimester of pregnancy has not been associated with adverse effects in the fetus. Reports on use of GBCAs during the second or third trimester are not rare, underscoring the usefulness of these agents in diagnosing various conditions. No harm to the fetus has been documented in these circumstances. ${ }^{7}$ No effects on embryo or fetal development were observed in rats or rabbits at doses up to $10 \mathrm{mmol} / \mathrm{kg} /$ day (in rats) or $3 \mathrm{mmol} / \mathrm{kg} /$ day (in rabbits). The doses in rats and rabbits were, respectively, 16 and 10 times the recommended human dose based on the body surface area. It is unknown whether Dotarem ${ }^{\circledR}$ crosses the human placenta, but other GBCAs do cross the placenta in humans and result in fetal exposure; therefore Dotarem ${ }^{\circledR}$ should be used during pregnancy only if the potential benefit outweighs the potential risk to the fetus. Current radiology recommendations discourage the use of GBCAs during pregnancy because their safety for the fetus has not been proven. Yet, available evidence suggests it is unlikely that these compounds have an adverse effect on the developing fetus; therefore, their use should not be limited, particularly given the important clinical reasons for MRI examinations during pregnancy. In conclusion, gadolinium use should be considered when the diagnostic study is important for the health of the mother. ${ }^{7}$

Concerning effects on lactation, it is not known whether Dotarem $^{\circledR}$ is excreted in human milk. Limited case reports on use of GBCAs in nursing mothers reports that $0.01 \%-0.04 \%$ of maternal gadolinium dose is excreted in human breast milk; absorption of gadolinium via the gastrointestinal tract is poor. Indeed, only tiny amounts of GBCAs given to a lactating mother reach the milk, and only a minute proportion entering the baby's gut is absorbed. The very small potential 
risk associated with absorption of the contrast medium may be considered insufficient to warrant stopping breast-feeding for 24 hours following the administration of GBCAs. ${ }^{8}$

The safety and efficacy of Dotarem ${ }^{\circledR}$ at a single dose of $0.1 \mathrm{mmol} / \mathrm{kg}$ have been established in pediatric patients from 2 years to 17 years of age. No dosage adjustment according to age is necessary in this population. For pediatric patients less than 2 years and more than 4 weeks old, after careful evaluation, the recommended dose should not exceed $0.1 \mathrm{mmol} / \mathrm{kg}$ $(0.2 \mathrm{~mL} / \mathrm{kg})$. Repeated administrations of GBCAs, close in time, are not recommended in pediatric patients, as well as their use for MRA in children under the age of 18 years.

For renal impaired patients, no Dotarem ${ }^{\circledR}$ dosage adjustment is recommended, and gadoteric acid can be removed from the body by hemodialysis (Figure 2).

\section{Elimination}

Dotarem $^{\circledR}$ is cleared from the body by urinary excretion. The half-life of gadoteric acid is about 1.4 hours in females, 2 hours in males, 5.1 hours in moderate renal impairment, and 13.9 hours in severe renal impairment.

Following a $0.1 \mathrm{mmol} / \mathrm{kg}$ dose of Dotarem ${ }^{\circledR}$, total gadolinium is excreted primarily in the urine, with $72.9 \% \pm 17.0 \%$ and $85.4 \% \pm 9.7 \%$ (mean $\pm \mathrm{SD}$ ) eliminated within 48 hours in female and male subjects, respectively.

\section{Imaging applications \\ Conventional and advanced contrast-enhanced magnetic resonance imaging (CE-MRI) of gadoteric acid}

\section{Neuroimaging}

Dotarem $^{\circledR}$ is a GBCA indicated for MRI of the brain (intracranial), spine, and associated tissues in adult and pediatric patients (2 years of age and older) to detect and visualize areas with disruption of the BBB and/or abnormal vascularity. MRI visualization of normal and pathological

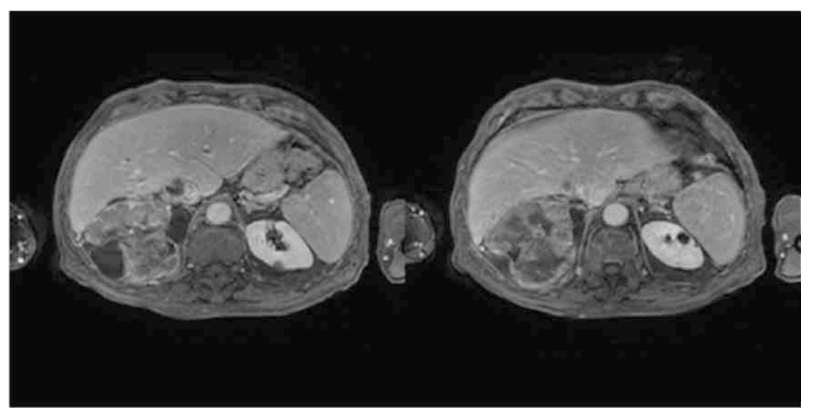

Figure 2 Contrast-enhanced TI High-Res Isotropic Vol Excitation axial images for the characterization of renal mass in a patient with severe renal impairment. tissue depends in part on variations in the radio frequency signal intensity that occurs with differing proton density and longitudinal/transverse relaxation times; GDCAs lead to a distinct shortening of proton relaxation times in plasma and may improve tissue visualization.

Gadoteric acid does not cross the intact BBB and, therefore, it does not enhance normal brain or lesions that have a normal BBB (eg, cysts, mature postoperative scars). However, disruption of the $\mathrm{BBB}$ or abnormal vascularity allows the distribution of gadoteric acid in lesions such as neoplasms, abscesses, and infarcts. Intravenously injected Gd-chelates are widely used to improve the efficacy of MRI in clinical practice and to identify, most notably, primary and metastatic brain neoplastic disease. Gd-chelates are extracellular, nontissue-specific, non-protein-binding, water-soluble compounds prepared at a concentration of 0.5 or $1 \mathrm{~mol} / \mathrm{L}$ of highly concentrated Gd-chelate agents (Figures 3 and 4A-D). ${ }^{9}$

\section{CE-Perfusion MRI}

Gd-chelate agents are used for several new indications such as MRA and perfusion imaging. Advanced functional MRI techniques (3-T MRI scan), such as dynamic, contrast-enhanced, perfusion-weighted imaging (PWI), utilize contrast agents to convey physiological information regarding the hemodynamics and neoangiogenic status of the lesion that is often complementary to anatomical information obtained through conventional imaging. Functional CE-MRI techniques such as PWI are in routine use for neuroimaging, most notably

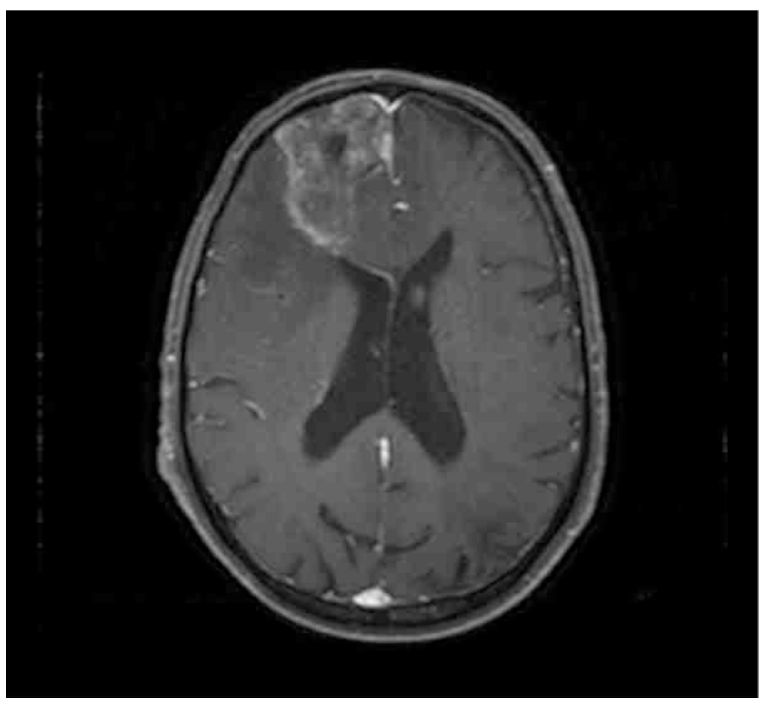

Figure 3 Patient affected by gliobastoma multiforme with renal impairment $\left(\right.$ GFR $=37 \mathrm{~mL} / \mathrm{min}$ ) who underwent a cerebral CE-MRI with Dotarem ${ }^{\circledR}$ at $3 \mathrm{~T}$ scan (axial TI weighted image).

Abbreviations: GFR, glomerular filtration rate; CE-MRI, contrast-enhanced magnetic resonance imaging. 

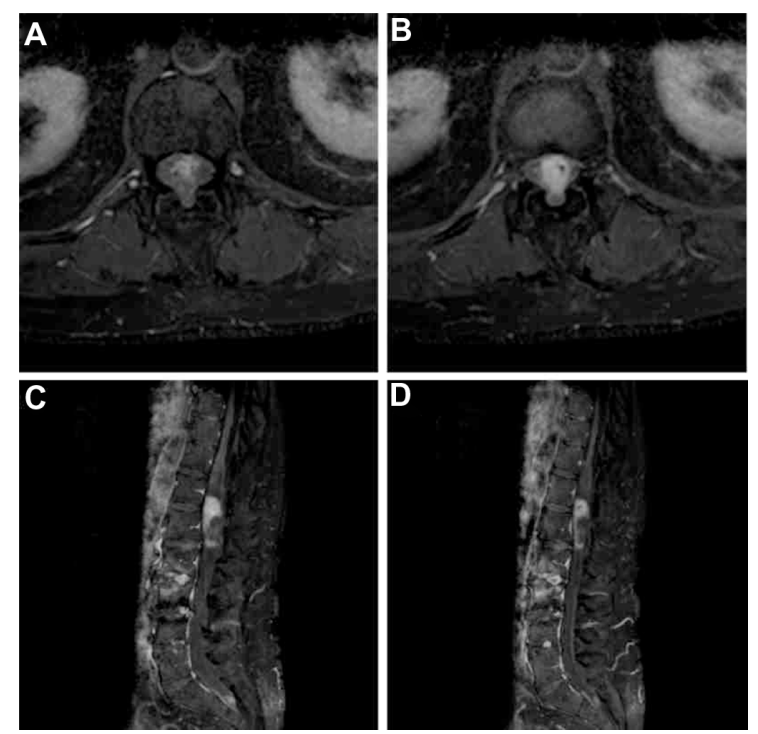

Figure 4 Axial (A and B) and sagittal (C and D) CE-TI-weighted images of the dorsolumbar spine in a patient affected by ependymoma of the intradural filum terminale.

for the evaluation and grading of brain lesions. Together with diffusion-weighted imaging, it allows assessing the eligibility of acute ischemic stroke patients for revascularization. The most common application of CE-MRI is in the evaluation of primary and secondary brain tumors. The goals of conventional brain tumor imaging are the sensitive detection and accurate depiction of disease in order to make a correct diagnosis and to provide, where appropriate, the tumor grade. The results of contrast-enhanced neuroimaging examinations provide a detailed description of the tumor's morphology, definition of the lesion borders, lesion enhancement, and the presence or absence of hemorrhage, necrosis, and mass effect, all of which are potentially related to tumor aggressiveness. In addition, conventional imaging can aid in the location of any adjacent critical structures before neurosurgery or radiotherapeutic intervention. MRI of brain lesions also plays a vital role during the posttherapeutic or intervention phase in determining treatment response: early identification of the lack of treatment efficacy can facilitate selection of an alternative therapeutic approach, potentially improving patient outcome. ${ }^{10}$ Brain tumor enhancement relies on two major conditions: first, a high vascular density, which is particularly present in most brain tumors; and, second, a rupture of the BBB. The application of small amounts of cyclic GDCAs is recommended, especially in patients with impaired renal function. ${ }^{11}$

One of the common strategies for increasing MRI sensitivity in the detection of brain lesions is increasing the dose of the contrast agent. While a standard dose of a GDCA is considered to be $0.1 \mathrm{mmol} / \mathrm{kg}$ body weight, many studies have demonstrated improved diagnostic performance with double $(0.2 \mathrm{mmol} / \mathrm{kg})$ or even triple $(0.3 \mathrm{mmol} / \mathrm{kg})$ doses. ${ }^{10}$ Before NSF was discovered, the safety profile of CE-MRI with GDCAs was considered as excellent, and, therefore, the use of higher doses became routine clinical practice when determination of the disease extent was the main goal of the examination. However, higher doses of the contrast agent increased the cost of MRI and were associated with potentially more falsepositive results. Since then, there has been increasing concern for the development of NSF in renal-impaired patients when higher doses are used. ${ }^{10}$

An alternative approach to improving diagnostic performance is the use of a contrast agent with higher relaxivity such as gadobenate dimeglumine (MultiHance ${ }^{\circledR}$, Bracco, Milan, Italy), gadopentetate dimeglumine (Magnevist ${ }^{\circledR}$ ), gadoteric acid $\left(\right.$ Dotarem $\left.^{\circledR}\right)$, gadodiamide (OmniScan ${ }^{\mathrm{TM}}$ ), and gadofosveset trisodium (Vasovist ${ }^{\circledR}$, Bayer Schering Pharma AG). ${ }^{10}$

Recent studies have shown a major advantage of macrocyclic gadolinium compounds as compared to linear gadolinium compounds in terms of stability, because the former group of agents is associated with a substantially lower amount of released, or free, gadolinium. ${ }^{11}$ Gadoteric acid $\left(\right.$ Dotarem $\left.^{\circledR}\right)$ and gadobutrol $\left(\right.$ Gadovist $\left.^{\circledR}\right)$ are both macrocyclic non-protein-binding gadolinium compounds that are approved and used in Europe and the United States. Although most gadolinium compounds (including gadoteric acid) have a gadolinium concentration of $0.5 \mathrm{mmol} / \mathrm{mL}$, Gadovist ${ }^{\circledR}$ has a gadolinium concentration of $1.0 \mathrm{mmol} / \mathrm{mL}$. In some reports, in vitro measurements of relaxivities at 1.5 and $3 \mathrm{~T}$ revealed $\sim 1.5$-fold higher relaxivity of gadobutrol compared to gadoteric acid. On the basis of these relaxivity data, it is hypothesized that $1.0 \mathrm{mmol} / \mathrm{mL}$ gadobutrol may allow a significantly lower dosage compared with $0.5 \mathrm{mmol} / \mathrm{mL}$ non-protein-binding gadolinium compounds to achieve equivalent efficacy in brain tumor assessment. ${ }^{11}$ However, to date, there is no data supporting any of these assumption (Figure 5).

\section{MR angiography}

Gadoteric acid is also used in MRA for study of supraaortic vessels, and CMR is employed in the detection of chronic myocardial infarctions.

MRA is a noninvasive and reliable tool for imaging blood vessels. The combination of a gadolinium-bolus subtraction technique and fast gradient-echo sequence has been shown to provide more accurate angiograms of the aortoiliac arteries and arteries of the lower extremities. ${ }^{12}$ The superiority of 


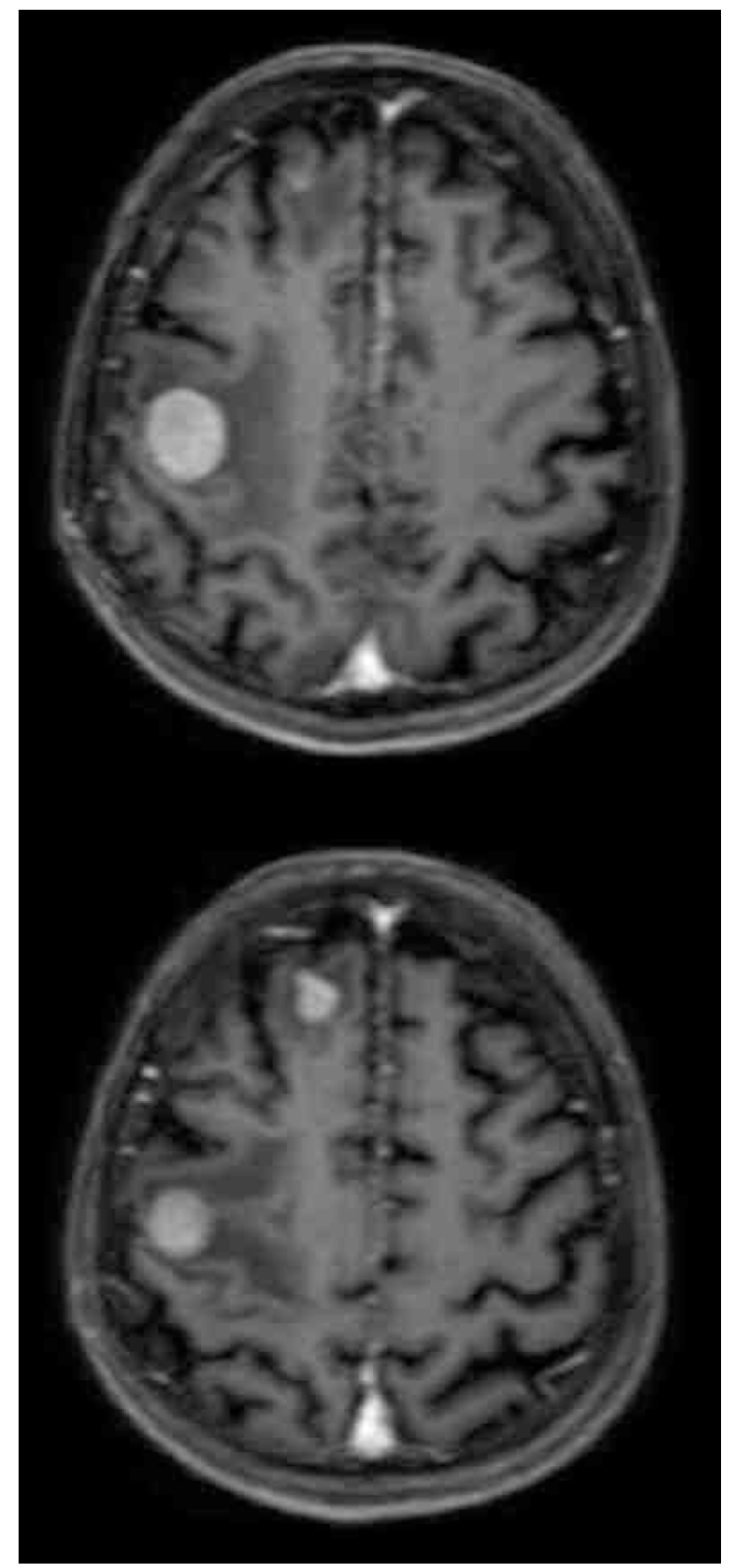

Figure 5 Axial CE-TI weighted image after iv administration of gadoteric acid in a patient with renal impairment and cerebral metastasis on right frontal lobe (3-T scan).

contrast-enhanced techniques as compared to unenhanced techniques, including the well-established time-of-flight (ToF) MRA technique, has to be reassessed taking into account the latest software and MR equipment. Two studies, were performed at 1.5 and 3.0 T, and Shah and Lim suggested that the CE-MRA of non-coronary arteries using Dotarem ${ }^{\circledR}$ provided better sensitivity, specificity, and image quality as compared to the ToF technique (Figure 6). ${ }^{12}$

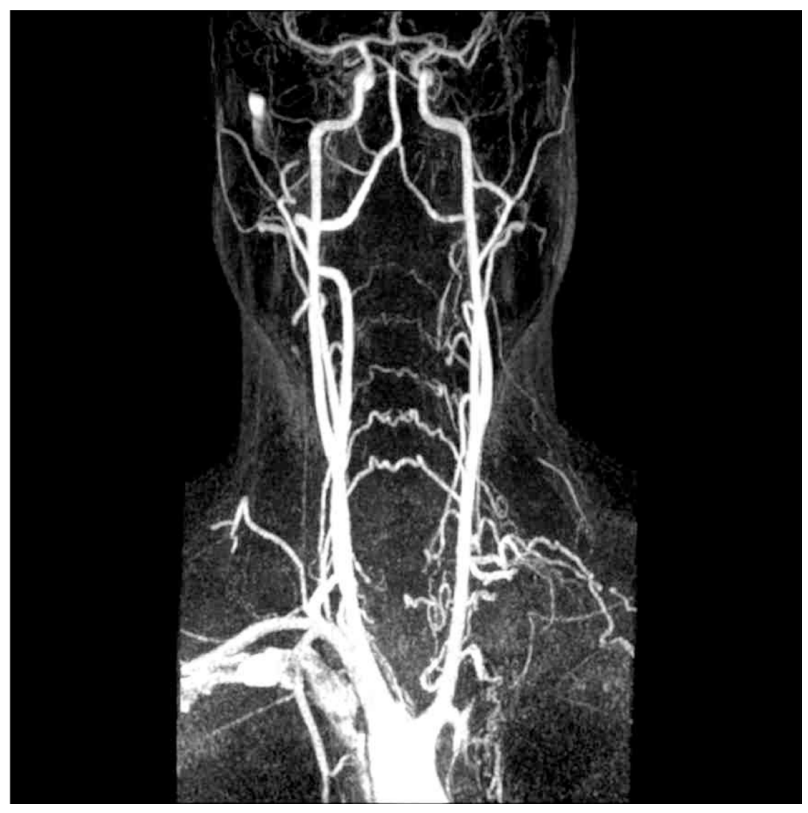

Figure 6 Maximum intensity projection reconstructed image of contrast-enhanced magnetic resonance angiography of epiaortic vessels.

\section{Cardiac MRI}

Cardiac MRI is a well-established modality in the diagnosis of myocardial infarction because this disease can be difficult to diagnose clinically. Moreover, biochemical evidence of infarction is present only for a limited time, and most myocardial infarctions are not associated with electrocardiography (ECG) Q-wave formation. MRI is of limited access and needs long acquisition times, preventing its use in the positive diagnosis of acute coronary syndromes without ST wave elevation (non-ST segment elevation myocardial infarction, NSTEMI), which is particularly challenging. ${ }^{13}$ The CMR images are gated to the ECG and obtained during repeated breath-holds. Myocardial infarction is identified by late gadolinium enhancement, after iv administration of gadolinium chelates. The most used contrast agent gadopentetate dimeglumine (Magnevist ${ }^{\circledR}$ ) is also associated with an increased risk of NSF in patients with impaired renal function when compared to macrocyclic contrast agents such as gadobutrol $\left(\right.$ Gadovist $\left.^{\circledR}\right)$ and gadoteric acid $\left(\right.$ Dotarem $\left.^{\circledR}\right)$, which are considered to be safer than linear contrast agents with regard to the risk of NSF. Late gadolinium-enhanced CMR images are obtained with a segmented inversion-recovery, fast, low-angle shot sequence on contiguous short-axis imaging planes 15 minutes after iv of $0.15 \mathrm{mmol} / \mathrm{kg}$ gadopentetate dimeglumine or gadoteric acid (in the absence of regulatory approval, this indication is performed under off-label use, so the optimal dose is not well established. However, most studies use between $0.10 \mathrm{mmol} / \mathrm{kg}$ and $0.20 \mathrm{mmol} / \mathrm{kg}$. 


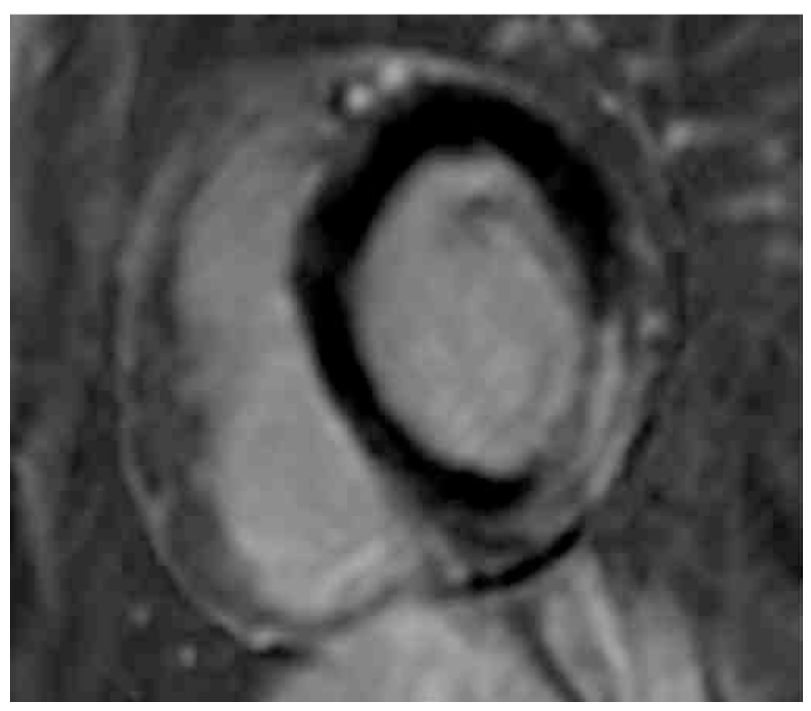

Figure 7 Late-enhancement phase-sensitivity inversion recovery short axis image of post infarction scar in the lateral-basal region of left ventricle.

Thus, it may be more appropriate to write "approximately $0.15 \mathrm{mmol} / \mathrm{kg}$ "). Cine CMR images can also be used to assess the contractility of the myocardium (Figures 7 and 8). ${ }^{14}$

\section{Pediatric imaging}

Gadoteric acid is the only macrocyclic and ionic GBCA commercially available. It has been approved by the US Food and Drug Administration, and it is indicated for intravenous use with MRI of the brain, spine, and associated tissues in adult and pediatric patients (2 years of age and older) to detect and visualize areas with disruption of the BBB and/or abnormal vascularity. For all the pediatric patients, the recommended dose is consistent with the currently proposed pediatric dose in the US of $0.1 \mathrm{mmol} / \mathrm{kg}$

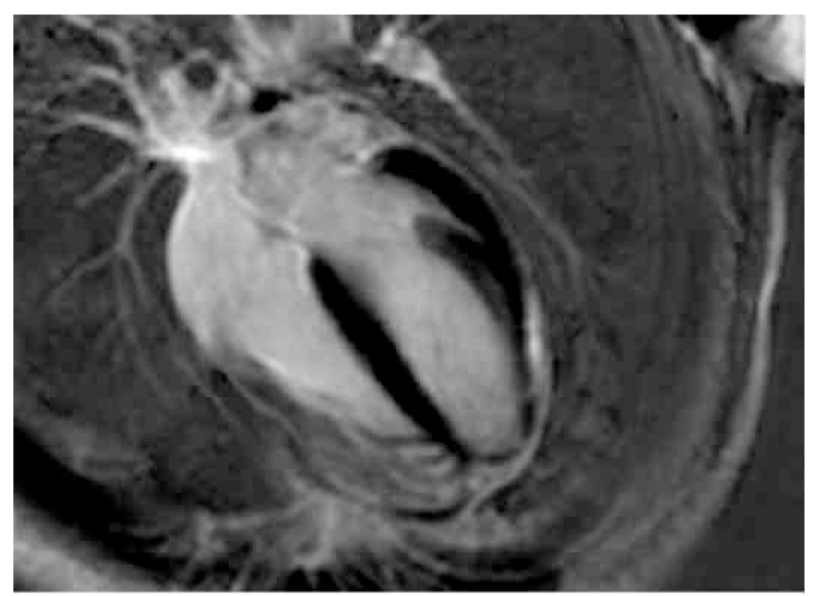

Figure 8 Late-enhancement phase-sensitivity inversion recovery long axis image of postinfarction scar in lateral-basal region of left ventricle.
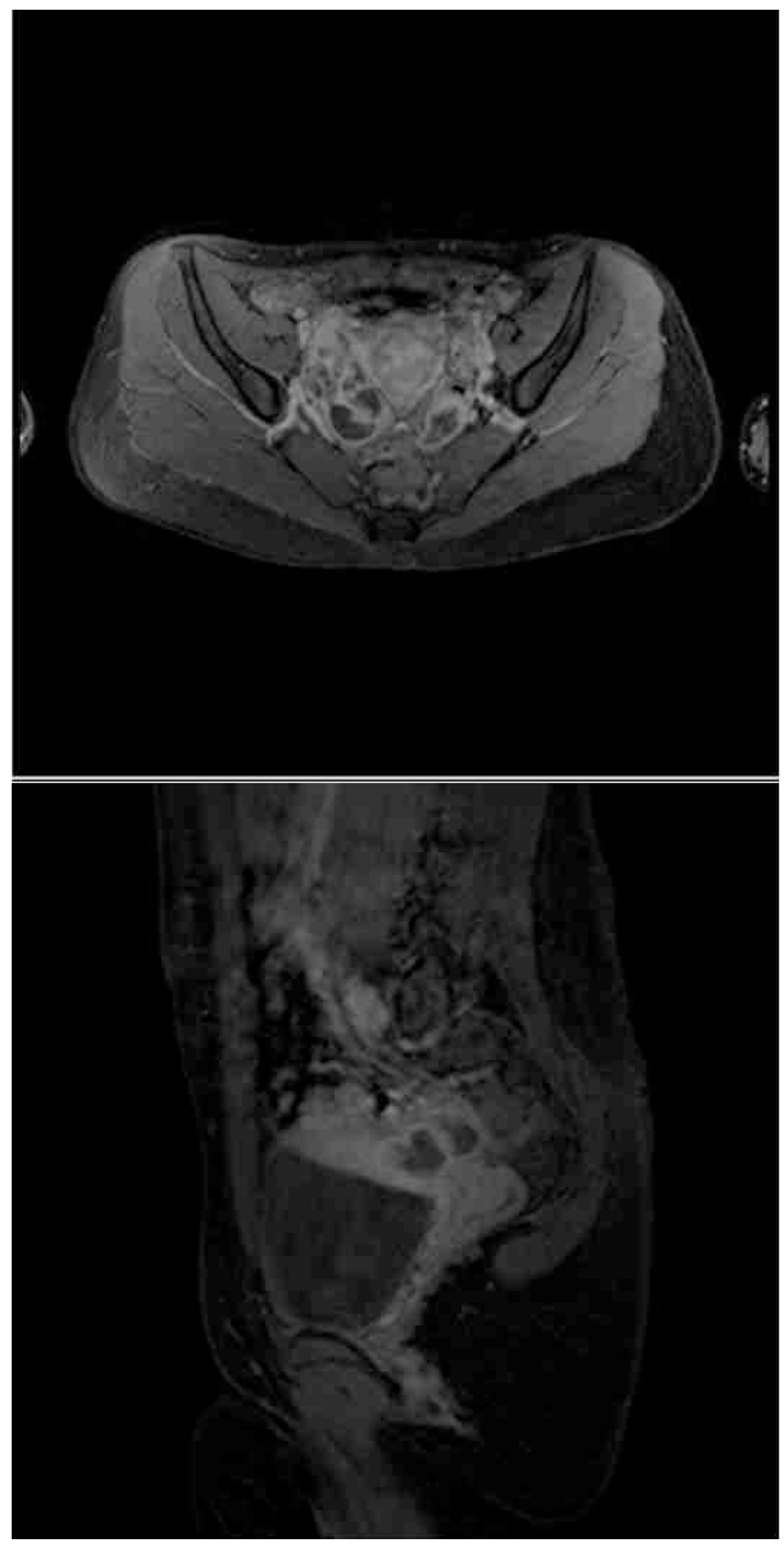

Figure 9 Axial and sagittal images contrast-enhanced TI High-Res Isotropic Vol Excitation of pelvis in a female pediatric patient with bilateral sactosalpinx.

body weight (ie, $0.2 \mathrm{~mL} / \mathrm{kg}$ ) administered as an iv bolus injection, manually or by a power injector, at a flow rate of approximately $2 \mathrm{~mL} / \mathrm{s}$ for adults and $1-2 \mathrm{~mL} / \mathrm{s}$ for children (Figure 9).

\section{Body MRI}

Dotarem $^{\circledR}$ is also used for body CE-MRI, particularly for imaging of breast and pancreas and for all patients with renal impairment who cannot undergo CE-MRI scan with higher concentrated contrast agents.

Breast MRI is a well-consolidated diagnostic technique, with precise indications in the breast cancer field. The use of 
a paramagnetic contrast medium allows the study of breast mass vascularization and tumor neoangiogenesis. This finding allows mass characterization not strictly based on morphological criteria but also on the qualitative and quantitative evaluation of its vascularization. Using this approach, breast MRI has been proven to have high sensitivity, up to $92 \%$, in characterizing breast masses, even though its specificity is variable. Breast MRI is performed using a 1.5-T system with a specific surface coil for breast examination. ${ }^{15}$ The following sequences are usually performed: sagittal TSE STIR; coronal FFE T1 3D breast Dyn acquired with six dynamic sequences, one before and five after iv bolus administration $(2 \mathrm{~mL} / \mathrm{s})$ of $0.1 \mathrm{mmol} / \mathrm{kg}$ of gadoteric acid followed by $10 \mathrm{~mL}$ of sterile saline solution; acquisition after contrast medium usually performed following a 10-second delay, each sequence lasts 1 minute. DWI is performed using an axial single-shot echoplanar imaging sequence centered on the lesions. ${ }^{16}$

The pictures obtained from the dynamic study are postprocessed using a dedicated appliance with the following procedures: automatic image subtraction, morphological qualitative analysis on enhancement foci, and quantitative and kinetic analysis of enhancement. Rounded regions of interest (ROI) are placed on the foci of enhancement identified on the subtracted images to measure the volumes on which the software generated the signal intensity time-curves. ${ }^{15}$

Typical maximum intensity projections (MIPs) obtained from postprocessing of subtracted images reveal not only the presence of enhanced lesions but also the angiographic vascular map of vessels of the whole breast. Vascular mapping with CE-MRI is a new, intriguing topic with demonstrated implications for the diagnosis of invasive breast cancer. To date, no study of a large series of pure in situ cancers has been undertaken. Angiogenesis imaging may be useful for differentiating invasive from noninvasive cancers. Its possible usefulness for the evaluation of the effect of neoadjuvant chemotherapy on locally advanced cancers and for the stratification of risk of disease deserves careful evaluation in future studies. Differentiation between arterial and venous breast vessels could be another area to be investigated. The use of high-relaxivity contrast agents such as gadobenate dimeglumine may provide real advantages for all of these future studies. ${ }^{17}$

The encouraging diagnostic yield of MRI in the detection and characterization of breast lesions must be weighed against its considerable costs. The possible role as a screening tool for women at increased risk for breast cancer must be defined in context with other less expensive modalities. In the meantime, the high sensitivity of dynamic contrast-enhanced MR imaging (DCE-MRI) should be studied in those patients at high risk for mammographically occult breast cancer, and in patients with already proven cancer when multifocality needs to be excluded prior to breast conservation therapy. Furthermore, young premenopausal women with a positive family history as well as patients with breast implants should be considered candidates for breast MRI (Figures 10 and 11). ${ }^{18}$

Dotarem $^{\circledR}$ can be used for the study of undetermined ovarian mass. In the developed world, ovarian cancer is the fifth commonest cancer affecting women. MRI has demonstrated superior specificity of approximately $88 \%$ for the diagnosis of adnexal lesions, achieved by observing the morphology of the lesion, detecting other features of malignancy such as ascites, and using early enhancement of malignant lesions as a discriminatory feature. ${ }^{19}$

In several other malignancies, including breast, prostate, and renal cancer, quantitative analysis of the dynamic contrast-enhanced sequence has proven useful. DCE-MRI has also been shown to correlate with tumor angiogenesis biomarkers in ovarian cancer.

In the study by Dilks et al, pelvic MRI was performed on a 1.5-T MRI unit. ${ }^{19}$ A pelvic phased array coil was used in all patients. An anti-peristaltic agent was administered by iv injection (20 mg hyoscine butylbromide, Buscopan; Boehringer Ingelheim, Germany). The following sequences were applied: axial T1-weighted spin-echo MRI from the renal hilum to the symphysis pubis; axial T2-weighted fast spin-echo (FSE) MRI of the pelvis or beyond if necessary to cover the larger adnexal masses; and sagittal T2-weighted FSE imaging from one femoral head to the other. Unenhanced and enhanced fat-suppressed,

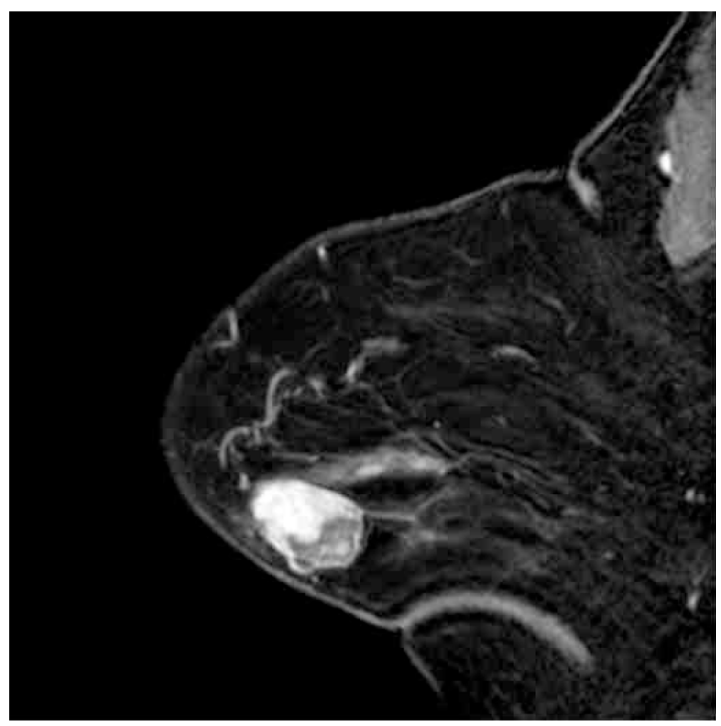

Figure 10 Bilateral breast imaging in the sagittal view with SENSE (BLISS) sagittal images of neoplastic lesion of right breast after iv administration of gadoteric acid. 


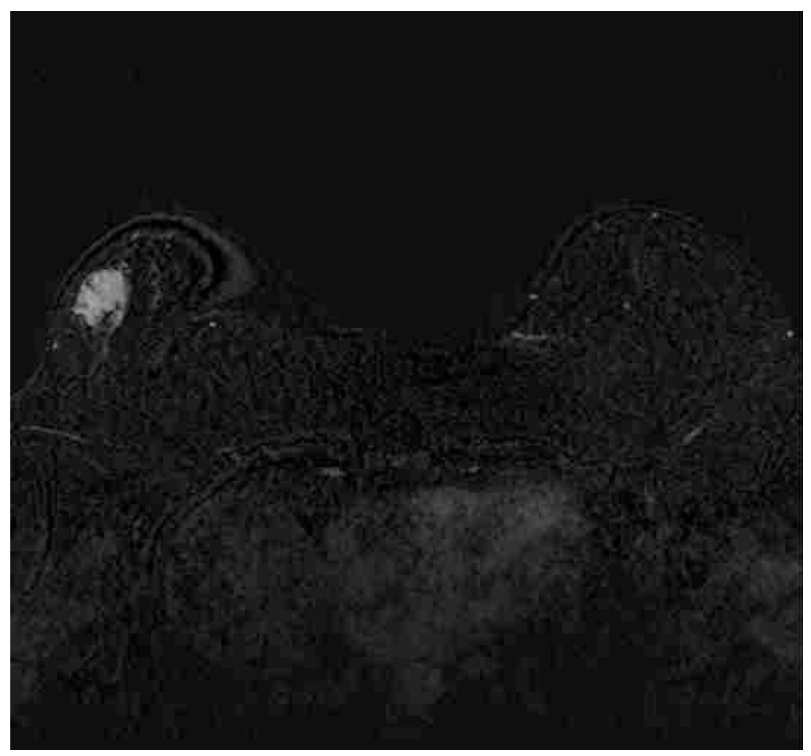

Figure I I Subtracted axial contrast-enhanced TI High-Res Isotropic Vol Excitation images with gadoteric acid.

spoiled, gradient-echo T1-weighted imaging was performed in the sagittal or axial plane. ${ }^{19}$ Contrast-enhanced images were obtained after iv injection of $0.1 \mathrm{mmol} / \mathrm{kg}$ of gadoteric acid $\left(\right.$ Dotarem $\left.^{\circledR}\right)$. The total acquisition time for this sequence was 22-30 seconds. This sequence was performed before and immediately after controlled pump iv injection of $3 \mathrm{~mL} / \mathrm{s}$ and then repeated at 30 seconds, 60 seconds, 90 seconds and 120 seconds into the examination. ${ }^{19}$

Multiparametric MRI has emerged as a safe, robust, and fairly reliable technique for the assessment of various pancreatic diseases. Major clinical indications for MR examinations of the pancreas include acute and chronic pancreatitis, analysis of cystic masses, assessment of lymph node size and infiltration, and staging of pancreatic carcinoma. Dynamic images are collected before and after the bolus administration of gadoteric acid at a dose of $0.1 \mathrm{mmol} / \mathrm{kg}$ during arterial, portal venous, and venous phases. The agent was administered with an automated injector at a rate of $1.0 \mathrm{~mL} / \mathrm{s}^{20}$

MRI examinations of the pancreas were performed at $1.5 \mathrm{~T}$ with multichannel phased-array coils. Patients were imaged in the supine position. No bowel preparation was used before MR imaging, and the patients did not receive any antispasmodic agent. Axial T2-weighted MR images were obtained with a free-breathing, fat-attenuated, FSE or turbo spin-echo (TSE) sequence. ${ }^{21}$

A transverse breath-hold three-dimensional (3D), fatattenuated, T1-weighted gradient-echo sequence (3D VIBE, LAVA, or 3D THRIVE) was performed before and four to six times after the iv administration of $0.1 \mathrm{mmol}$ of gadoteric acid per kilogram of body weight followed by a $20-\mathrm{mL}$ saline flush, at an injection rate of $2 \mathrm{~mL} / \mathrm{s}$. The first acquisition was obtained 25-30 seconds after the administration of contrast material, and the second acquisition at 60 seconds. The third acquisition was obtained 120 seconds after injection, and the final acquisition (equilibrium phase) was performed 4 minutes after the beginning of administration of the contrast material. All acquisitions were performed during suspended respiration at end expiration. ${ }^{21}$

Pancreatic CE-MRI makes it possible to obtain an excellent overview of the anatomy in the upper abdomen, but it also assists in determining loco-regional infiltration of pancreatic masses and lymph node involvement and thus justifies its integration into a standard protocol. ${ }^{20}$

\section{Conclusion}

In conclusion, gadoteric acid is a handy contrast medium for total body applications, in both adults and pediatric patients. In patients with renal impairment, gadoteric acid can be used with an acceptable degree of safety. Due to its versatility, gadoteric acid could be considered a kind of "passe-partout" in clinical practice.

\section{Disclosure}

The authors declare no conflicts of interest in this work.

\section{References}

1. Le Mignon MM, Chambon C, Warrington S, Davies R, Bonnemain B. Gd-DOTA. Pharmacokinetics and tolerability after intravenous injection into healthy volunteers. Invest Radiol 1990;25(8):933-937.

2. Morcos SK, Thomsen HS. Nephrogenic systemic fibrosis: more questions and some answers. Nephron Clin Pract. 2008;110:c24-c31.

3. Penfield JG, Reilly RF. Nephrogenic systemic fibrosis risk: is there a difference between gadolinium-based contrast agents? Semin Dial. 2008;21(2):129-134.

4. Ledneva E, Karie S, Launay-Vacher V, Janus N, Deray G. Renal safety of gadolinium-based contrast media in patients with chronic renal insufficiency. Radiology. 2009;250(3):618-628.

5. Attenberger UI, Runge VM, Morelli JN, Williams J, Jackson CB, Michaely HJ. Evaluation of gadobutrol, a macrocyclic, nonionic gadolinium chelate in a brain glioma model: comparison with gadoterate meglumine and gadopentetate dimeglumine at $1.5 \mathrm{~T}$, combined with an assessment of field strength dependence, specifically 1.5 versus $3 \mathrm{~T}$. J Magn Reson Imaging. 2010;31(3):549-555.

6. Maurer M, Heine O, Wolf M, Durmus T, Wagner M, Hamm B. Tolerability and diagnostic value of gadoteric acid in the general population and in patients with risk factors: results in more than 84,000 patients. Eur J Radiol. 2012;81(5):885-890.

7. Garcia-Bournissen F, Shrim A, Koren G. Safety of gadolinium during pregnancy. Can Fam Physician. 2006;52:309-310.

8. Webb JA, Thomsen HS, Morcos SK. Members of contrast media safety committee of European Society of urogenital radiology (ESUR), the use of iodinated and gadolinium contrast media during pregnancy and lactation. Eur Radiol. 2005;15(6):1234-1240.

9. Tombach B, Heindel W. Value of 1.0-M gadolinium chelates: review of preclinical and clinical data on gadobutrol. Eur Radiol. 2002;12(6): $1550-1556$. 
10. Giesel FL, Mehndiratta A, Essig M. High-relaxivity contrast-enhanced magnetic resonance neuroimaging: a review. Eur Radiol. 2010;20(10): 2461-2474.

11. Kramer H, Runge VM, Naul LG, Loynachan AT, Reiser MF, Wintersperger BJ. Brain MRI with single-dose $(0.1 \mathrm{mmol} / \mathrm{kg})$ Gadobutrol at $1.5 \mathrm{~T}$ and $3 \mathrm{~T}$ : comparison with $0.15 \mathrm{mmol} / \mathrm{kg}$ gadoterate meglumine. AJR Am J Roentgenol. 2010;194:1337-1342.

12. Shah SH, Lim T-H. Evaluation of meglumine gadoterate-enhanced MR angiography (MRA) compared with time-of-flight MRA in the diagnosis of clinically significant non-coronary arterial disease: a pooled analysis of data from two clinical trials. Br J Radiol. 2012;85(1013):596-605.

13. Laissy JP, Gaxotte V, Ironde-Laissy E, et al. Cardiac diffusion-weighted MR imaging in recent, subacute, and chronic myocardial infarction: a pilot study. J Magn Reson Imaging. 2013;38:1377-1387.

14. Ichikawa Y, Sakuma H, Suzawa N, et al. Late gadolinium-enhanced magnetic resonance imaging in acute and chronic myocardial infarction. Improved prediction of regional myocardial contraction in the chronic state by measuring thickness of nonenhanced myocardium. J Am Coll Cardiol. 2005;45(6):901-909.

15. Caproni N, Marchisio F, Pecchi A, et al. Contrast-enhanced ultrasound in the characterisation of breast masses: utility of quantitative analysis in comparison with MRI. Eur Radiol. 2010;20:1384-1395.
16. Pereira FP, Martins G, Figueiredo E, et al. Assessment of breast lesions with diffusion-weighted MRI: comparing the use of different $\mathrm{b}$ values. AJR Am J Roentgenol. 2009;193(4):1030-1035.

17. Sardanelli F, Fausto A, Menicagli L, Esseridou A. Breast vascular mapping obtained with contrast-enhanced MR imaging: implications for cancer diagnosis, treatment, and risk stratification. Eur Radiol. 2007;17(Suppl 6):F48-F51.

18. Kacl GM, Liu P-F, Debatin JF, Garzoli E, Caduff RF, Krestin GP. Detection of breast cancer with conventional mammography and contrast-enhanced MR imaging. Eur Radiol. 1998;8:194-200.

19. Dilks P, Narayanan P, Reznek R, Sahdev A, Rockall A. Can quantitative dynamic contrastenhanced MRI independently characterize an ovarian mass? Eur Radiol. 2010;20:2176-2183.

20. Herborn CU, Vogt FM, Lauenstein TC, Goyen M, Debatin JF, Ruehm SG. Evaluation of steady state free precession imaging of the pancreas. Eur Radiol. 2005;15:1629-1633.

21. Guerrache Y, Soyer P, Dohan A, et al. Solid-pseudopapillary tumor of the pancreas: MR imaging findings in 21 patients. Clin Imaging 2014;38(4):475-482.
Reports in Medical Imaging

\section{Publish your work in this journal}

Reports in Medical Imaging is an international, peer-reviewed, open access journal publishing original research, reports, reviews and commentaries on all areas of medical imaging. The manuscript management system is completely online and includes a very quick and fair peer-review system, which is all easy to use.

\section{Dovepress}

Visit http://www.dovepress.com/testimonials.php to read real quotes from published authors. 